\title{
Aprendendo com o Imprevisível: Saúde Mental dos Universitários e Educação Médica na Pandemia de Covid-19
}

\author{
Learning from the Unpredictable: College Students' Mental Health and Medical Education in the Covid-19 Pandemic
}

\author{
Bráulio Brandão Rodrigues ${ }^{\circledR}$, Rhaissa Rosa de Jesus Cardoso' \\ Caio Henrique Rezio Peres ${ }^{\perp}$, Fábio Ferreira Marques' ${ }^{(\mathbb{B}}$
}

\section{RESUMO}

Introdução: A pandemia causada pelo novo coronavírus (Covid-19) promoveu mudanças em todo o mundo, gerando modificações na estrutura organizacional do ensino superior. A educação médica teve que suspender atividades presenciais e estágios práticos, e adotar a metodologia de ensino a distância e avaliações on-line para os discentes de Medicina.

Objetivo: Discorrer sobre o impacto da pandemia na saúde mental dos universitários e na educação médica.

Método: Trata-se de uma revisão de literatura realizada nas seguintes bases de dados: Scientific Electronic Library Online (SciELO), Literatura Latino-Americana e do Caribe em Ciências da Saúde (LILACS) e Medical Literature Analysis and Retrieval System Online (MEDLINE). Utilizaram-se, na busca de artigos, os Descritores em Ciências da Saúde (DeCS): "saúde mental”, "pandemia", "educação superior", "estudantes" e "Covid-19". Foram considerados estudos com seres humanos e estudos de literatura publicados de 2018 até o momento do levantamento de dados.

Resultado: Nas bases de dados, encontraram-se 1.473 artigos que foram submetidos aos critérios de inclusão e exclusão. Desconsiderando a duplicidade em outras bases de dados, obtiveram-se 43 artigos, dos quais 31 foram utilizados nesta revisão de literatura. Tem-se um grande número de estudos experimentais sobre a educação superior que são úteis na disseminação de conhecimento e possibilidade de replicação. Os dados referentes à saúde mental dos estudantes universitários abordam aspectos sobre a presença de transtornos psiquiátricos relacionados à temática, como depressão, ansiedade, e estresse pós-traumático, a partir de testes de triagem diagnóstica, nas variações presencial e on-line.

Conclusão: Como os estudantes de Medicina apresentam incertezas sobre o futuro de sua formação em decorrência dessas transformações, são submetidos a uma carga emocional que causa/deflagra danos à saúde mental deles. Existem ainda dúvidas sobre os reflexos desse contexto no período "pós-Covid" e seus impactos na educação médica, assim como sobre a manutenção de medidas adotadas em tempos de crise.

Palavras-chave: Saúde Mental; Estudantes; Educação Superior; Pandemia; Covid-19.

\begin{abstract}
Introduction: The pandemic caused by the new coronavirus (Covid-19) has promoted changes worldwide, including changes in the organizational structure of higher education. Medical education has had to suspend face-to-face activities and practical internships, adopting distance learning methodology as well as online assessments for medical students.
\end{abstract}

Objective: To discuss the impact of the pandemic on university students' mental health and medical education.

Method: This is a review of the following databases: Scientific Electronic Library Online (SciELO), Latin American and Caribbean Literature in Health Sciences (LILACS) and Medical Literature Analysis and Retrieval System Online (MEDLINE). In the search for articles, the Health Sciences Descriptors (DeCS) used were: "mental health", "pandemic", "higher education", "students", "Covid-19". The studies considered were those which involved human beings, literature studies, and published between 2018 and the moment of the data collection.

Result: The 1,473 articles found in the databases were subjected to the inclusion and exclusion criteria, disregarding repeats in different databases, leaving 43 articles, from which the 31 articles used in this literature review were obtained. There are a large number of experimental studies on higher education, which are useful for disseminating knowledge and enabling replication. The data referring to the mental health of university students demonstrate the presence of psychiatric disorders related to the theme such as depression, anxiety, and post-traumatic stress, based on face-to-face and online diagnostic screening tests.

Conclusion: Medical students, under intense emotional burden, are feeling uncertain about the future of their training as a result of these transformations, reporting damage to their mental health. There are also doubts about the repercussions in this context in the "post-Covid" period and the impacts on medical education, as well as the maintenance of the measures which have been adopted during the crisis.

Keywords: Mental Health; Students; Higher Education; Pandemic; Covid-19.

'Centro Universitário de Anápolis, Anápolis, Goiás, Brasil.

Correspondência:

Bráulio Brandão Rodrigues.

Avenida das Araucárias, lote 4150, Blend Apartaments, bloco B, ap. 603, Águas Claras, Brasília, DF, Brasil. CEP: 71936-250.

E-mail: brandaobbr@gmail.com

Recebido em 11/08/20; Aceito em 04/09/20. 


\section{INTRODUÇÃO}

Em dezembro de 2019, foi relatado, em Wuhan, na China, um surto de doença causada pelo coronavírus da síndrome respiratória aguda severa 2 (severe acute respiratory syndrome coronavirus 2 - Sars-CoV-2), a coronavirus disease (Covid-19). Rapidamente, o vírus se espalhou pelo mundo todo, tornando-se a primeira pandemia por coronavírus ${ }^{1,2}$. Até o dia 9 de agosto de 2020, havia 19.462.112 casos confirmados e 722.285 mortes no mundo. No Brasil, na mesma data, havia 2.962.442 casos confirmados de Covid-19 e 99.572 mortes dela decorrentes ${ }^{3}$. No entanto, esses números podem ser ainda maiores, considerando casos positivos não testados e atrasos nas notificações ${ }^{4}$.

A alta virulência do novo coronavírus, associada à inexistência de um tratamento eficaz para a doença, levou à adoção de medidas emergenciais preventivas capazes de proteger a saúde e salvar vidas em todo o mundo, como a quarentena e o isolamento social ${ }^{5}$. A quarentena pode causar inúmeros sintomas psicopatológicos, como humor deprimido, irritabilidade, ansiedade, medo, raiva, insônia, sintomas de estresse pós-traumático, confusão, entre outros ${ }^{6}$. Na China, durante a fase inicial do surto de Covid-19, mais da metade dos entrevistados apontou, em um inquérito, que a intensidade do impacto psicológico varia de moderada a grave, e identificouse a ansiedade grave em um terço dos indivíduos. O impacto psicológico negativo foi maior em mulheres, estudantes e pessoas que tinham algum sintoma físico prévio?.

Alémdisso,porcontadasatuaiscondiçõesdequarentena, fecharam-se escolas e universidades, e suspenderam-se encontros presenciais de ensino e aprendizagem, o que afetou as fases fisiológicas da vida e induziu muitas preocupações individuais e coletivas ${ }^{8}$. No entanto, diferentemente de outros estudantes, os discentes de Medicina têm uma compreensão mais profunda da doença, deixando-os ainda mais ansiosos durante o período de quarentena. Além da ansiedade devido à doença em si, a pandemia contribuiu para o atraso na prática clínica dos estudantes, que estavam em quarentena, o que atrapalhou, significativamente, a programação acadêmica deles, deixando-os ainda mais ansiosos ou deprimido?.

Em virtude do fechamento das universidades, a educação mediada por tecnologias, como o Ensino a Distância (EaD), suscitou inúmeros debates sobre os rumos da educação na atualidade e no período pós-Covid, evidenciando novas oportunidades para a educação ${ }^{10}$. $O$ ensino on-line agora é uma nova rotina para muitos alunos, mas enfrenta grandes desafios, uma vez que nem todos têm acesso a esse tipo de educação, considerando a desigualdade social em muitas nações ${ }^{11}$. Embora o fechamento temporário de escolas como resultado de crises de saúde e de outras emergências não seja novo, infelizmente a escala e a velocidade globais da atual interrupção educacional são incomparáveis e, se prolongadas, podem causar dor e sofrimento psicológicos em diferentes níveis ${ }^{12}$.

Especialistas alertam que o impacto do Sars-CoV-2 repercutirá no ensino superior global por muito mais tempo depois que o surto for finalmente controlado. Nas áreas mais afetadas, as universidades enfrentam a perspectiva de perder um semestre inteiro ou até mais ${ }^{13}$. Diante do exposto, esta revisão tem o objetivo de destacar o impacto da pandemia na saúde mental dos universitários e na educação médica durante esse período.

\section{MÉTODO}

Trata-se de uma revisão de literatura com a finalidade de reunir e sintetizar o conteúdo de artigos e livros acerca do impacto da pandemia de Sars-CoV-2 na saúde mental dos universitários e na educação superior. Foram considerados estudos realizados com grupos de seres humanos e estudos de literatura publicados de 2018 até o momento do levantamento de dados. Esse recorte temático levou em conta o caráter atual do tema e o fato de a doença ter se disseminado amplamente pelo mundo no referido ano.

O levantamento bibliográfico foi realizado nas seguintes bases de dados: Scientific Electronic Library Online (SciELO), Literatura Latino-Americana e do Caribe em Ciências da Saúde (LILACS) e Medical Literature Analysis and Retrieval System Online (MEDLINE). Utilizaram-se, na busca de artigos, os Descritores em Ciências da Saúde (DeCS): "saúde mental", “pandemia”, "educação superior”, “estudantes" e "Covid-19". Empregaram-se os operadores booleanos "OR" e "AND" com o objetivo de aprimorar a seleção, assim como os correspondentes em inglês e espanhol dos descritores empregados.

Os critérios de inclusão utilizados foram: trabalhos científicos, nacionais e internacionais, relacionados à temática de estudo, envolvendo seres humanos, nos idiomas português, inglês ou espanhol, publicados nos últimos três anos, disponíveis on-line na forma de artigo completo e gratuito e com acesso a todos os públicos. Já os critérios de exclusão foram: artigos que não estivessem dentro do recorte temático escolhido, bem como resumos sem texto completos disponíveis gratuitamente, além de pesquisas com enfoque em outras temáticas fora dos descritores, após leitura prévia do resumo dos artigos.

\section{RESULTADO}

Encontraram-se 1.473 artigos nas seguintes bases de dados: SciELO $(n=137)$, LILACS $(n=931)$ e MEDLINE $(n=$ 405). Após a utilização dos critérios de inclusão e exclusão, considerando cada artigo apenas em sua primeira ocorrência (e não a duplicidade em outras bases de dados), e a leitura integral dos textos, obtiveram-se 43 artigos. Destes, foram 
selecionadas as publicações mais relevantes, restando apenas os 31 artigos utilizados nesta revisão de literatura.

Entre os artigos analisados, notou-se um grande número de publicações referente a países acometidos no início da pandemia e que, no momento, se encontram em declínio do número de casos infectados, como países europeus e a China, ou experiências em surtos/pandemias anteriores. Ressalta-se que boa parte dos estudos brasileiros é composta de experiências locorregionais e de estudos transversais.

Em relação aos dados sobre a educação superior e sua situação atual, também se observou um grande número de estudos experimentais, os quais são úteis a esta análise, no sentido de disseminação de conhecimento e de possibilidade de replicação em outros locais. Os dados referentes à saúde mental dos estudantes universitários abordam principalmente discentes da área da saúde, com um enfoque maior no curso de Medicina. Esses dados discorrem sobre a presença de transtornos psiquiátricos relacionados à temática, como depressão, ansiedade e estresse pós-traumático, a partir de testes de triagem diagnóstica, nas variações presencial e on-line.

\section{DISCUSSÃO}

O surto de Covid-19 levou a maioria dos países a optar por medidas de confinamento da população e distanciamento social como forma de diminuir a propagação do vírus ${ }^{14}$. Contudo, a quarentena é, muitas vezes, uma experiência desagradável para quem a experimenta, já que a distância dos entes queridos, o tédio, a incerteza sobre a real situação da doença e a perda de liberdade podem causar efeitos dramáticos ${ }^{15}$. Recentemente, foi publicado um estudo sobre pesquisas em saúde mental associadas ao surto de Covid-19 que tiveram como alvo diversas populações. Esse estudo mostrou que a prevalência de depressão e ansiedade era de $50,7 \%$ e $44,7 \%$, respectivamente 9 .

Os efeitos psicopatológicos, associados à quarentena e ao isolamento social, são agravados quando a pessoa possui algum antecedente psiquiátrico prévio, em especial a ansiedade, o que pode aumentar a incidência de estresse pós-traumático. Esses efeitos foram identificados tanto em anteriores experiências de isolamento6 quanto no atual cenário de pandemia ${ }^{6,7}$. Além disso, podem ocorrer em pessoas previamente sadias, por estarem relacionados aos seguintes aspectos: duração do período de isolamento social, medo de infecção, frustração e tédio, suprimentos inadequados, informações inadequadas, situação financeira/econômica e estigma sobre a situação vivenciada ${ }^{6}$.

Estudos sugerem que emergências de saúde pública podem ter muitos efeitos psicológicos em estudantes universitários, como ansiedade, medo, preocupação, entre outros $^{16}$. Por conta das intensas preocupações em torno da Covid-19, inúmeras universidades resolveram interromper as aulas presenciais e evacuar os alunos, o que contribui, ainda mais, para o surgimento de negativos efeitos psicológicos entre os estudantes. Alguns podem ter problemas com a solidão e o isolamento por causa da falta de contato com amigos e parceiros. Outros deixam de receber o aconselhamento de profissionais no campus universitário e, com isso, têm os seus sintomas psicológicos agravados, aumentando o risco de suicídio e o abuso de substâncias ${ }^{17}$.

Há ainda uma questão que envolve a incerteza sobre o efeito da pandemia: a maior preocupação dos estudantes em relação à própria formação e à possibilidade de eles encontrarem um emprego ou matricularem-se em futuros programas de estudos como a residência ${ }^{18}$. As interrupções dos projetos de pesquisa e estágios comprometem o cronograma de estudos, atrasam a graduação e prejudicam a competitividade no mercado de trabalho, o que, por sua vez, alimenta a ansiedade entre os estudantes universitários ${ }^{17}$. O fato é que os estudantes constituem uma população particularmente vulnerável a problemas de saúde mental em vista dos desafios comumente associados à transição para a vida adulta e das frequentes dificuldades econômicas e materiais desse grupo ${ }^{19,20}$.

Além disso, pelo fato de os jovens poderem ser portadores assintomáticos21, os estudantes têm preocupações e temores em relação à infecção e transmissão da Covid-19 a seus familiares mais idosos por representarem o grupo com maior risco de complicações por essa doença ${ }^{17}$. Esse estresse adicional, combinado aos efeitos da pandemia, pode ser visto como um maior risco para o desenvolvimento de problemas de saúde mental ${ }^{18}$.

Um estudo realizado em uma Faculdade de Medicina na China mostrou que a prevalência de depressão entre os estudantes foi de $35,5 \%$ e a prevalência de ansiedade chegou a $22,1 \%$, e a maioria apresentava um estado de depressão ou ansiedade leve ou moderada ${ }^{22}$. Com resultados semelhantes, outro estudo realizado na Faculdade de Medicina da Universidade King Saud identificou que $23,5 \%$ dos estudantes se sentiam desanimados ou deprimidos ${ }^{8}$. No entanto, pesquisas realizadas na China com estudantes de Medicina demonstraram que apenas uma pequena parcela dos discentes relatou ansiedade moderada $(2,7 \%)$ ou grave $(0,9 \%)$. Esse estudo também mostrou que morar com os pais estava associado a taxas muito mais baixas de ansiedade severa em estudantes, enquanto morar em áreas rurais, sem ter uma renda estável e conhecendo alguém infectado com Covid-19 aumentava o risco de ansiedade severa ${ }^{23}$.

Outro lado importante a ser destacado é que a pandemia 
causou perturbações significativas no ensino médico. Isso levou a uma incerteza generalizada e afetou todos os cantos do mundo. Embora muitas pesquisas e discussões envolvam ramificações políticas, financeiras e de saúde pública imediatas e potencialmente duradouras da pandemia da Covid-19, o diálogo sobre como isso afetou e afetará a educação médica está um pouco atrasado ${ }^{24-27}$.

O surto de Sars proporcionou uma oportunidade para introduzir e integrar ainda mais a tecnologia da informação nas metodologias de ensino e aprendizagem baseada em problemas (problem-based learning - PBL) nos cursos de Medicina $^{26}$. Um cenário comparável foi o surto de Sars no início dos anos 2000, que forçou o fechamento de escolas de Medicina em Hong Kong e no Canadá. Em resposta aos estágios cancelados, assim como o que ocorreu em 2020, muitas escolas de Medicina, durante esse período, fizeram a transição para aulas em vídeo e salas de bate-papo on-line para substituir experiências clínicas. Embora a simulação tenha fornecido oportunidades alternativas, percebeu-se uma falta de fidelidade e realismo com esses métodos de aprendizagem. Como resultado, esses estudantes de Medicina tiveram oportunidades reduzidas de aprendizado prático ${ }^{28,29}$.

Quando se discutem a educação on-line e o EaD, o coronavírus abre uma questão importante e urgente que afeta a saúde mental, já que são temas praticamente inexplorados e seus resultados ainda não foram validados. A educação online não se limita ao $\mathrm{EaD}$, pois se trata de um agrupamento de processos de ensino e aprendizagem realizados no ciberespaço. São circunstâncias inéditas e que geram estresse, favorecendo a angústia e a busca acirrada por novos conhecimentos. Pesquisadores destacam que a ansiedade e a depressão, exacerbadas pelas incertezas e pela intensificação do fluxo de informações, crescerão extensivamente. As consequências fisiológicas negativas do estresse se manifestarão com um impacto negativo na educação e, portanto, na dor e no sofrimento psicológico' ${ }^{12}$.

Ademais, os efeitos econômicos e na vida cotidiana, bem como atrasos nas atividades acadêmicas, estão relacionados a sintomas de ansiedade $(p<0,001)$ durante $o$ período de pandemia, conforme encontrado na literatura23. Outro estudo demonstrou que a maioria dos alunos $(56,2 \%$, $\mathrm{n}=530$ ) sentiu uma diminuição no tempo que eles passaram estudando e diminuição acentuada no desempenho, e o sexo feminino apresentou maior prevalência ${ }^{8}$. A transição para o online levantou questões para os professores sobre capacidade deles de lidar com a tecnologia existente. Além disso, muitas universidades não possuem infraestrutura ou recursos suficientes para facilitar o ensino on-line ${ }^{30,31}$.

Várias universidades suspenderam os exames finais do semestre, enquanto a avaliação continuará com as aulas online. A transição do ensino presencial para o on-line tem um sério impacto nas avaliações. Embora a tecnologia tenha sido usada anteriormente para apoiar o ensino e a aprendizagem, o aspecto da avaliação geralmente é subdesenvolvido. A aplicação de avaliações on-line nos cursos projetados para o aprendizado presencial é uma tarefa desafiadora. Os alunos, assim como os professores, não têm certeza sobre o procedimento para administrar tarefas, projetos e outras avaliações contínuas pendentes, além da dificuldade em monitorar como os alunos estão on-line e garantir que não trapaceiem durante os testes ${ }^{31,32}$.

\section{CONCLUSÃO}

A pandemia de Covid-19 promoveu inúmeras mudanças na sociedade, como o medo de contaminação, o isolamento social, a suspensão de atividades acadêmicas presenciais e a adoção de novas metodologias de ensino universitário. Os estudantes de Medicina se inserem nesse contexto com incertezas sobre o futuro de sua formação em decorrência dessas transformações. Permeados por toda carga emocional que o curso transporta, ainda devem lidar com mudanças na forma de ensinar, em uma velocidade acelerada.

Com isso, muitos transtornos mentais são deflagrados e/ ou desencadeados neste momento de instabilidade, tornando a saúde mental desses indivíduos o enfoque de vários estudos e do ensino superior. Ademais, a própria educação médica, inserida nesse contexto de pandemia, passa por um processo de transformação que deverá ser mais bem discutido. Existe ainda muita incerteza sobre os reflexos desse período no período "pós-Covid", os impactos futuros disso na educação médica e a manutenção de medidas adotadas em tempos de crise.

Dessa forma, gerenciar o estresse e bem-estar psicossocial é tão importante, neste momento, quanto cuidar da saúde física. Faz-se imprescindível, portanto, a adoção de estratégias, por parte das instituições de ensino superior, para amenizar o sofrimento psíquico dos estudantes. É fundamental que haja a criação ou o aprimoramento de núcleos de apoio psicossocial aos discentes de fácil acesso para que consigam cuidar de sua saúde mental no atual cenário. $O$ uso da tecnologia pode auxiliar não somente as aulas a distância, mas também esse apoio oferecido aos alunos, sendo possíveis atendimentos on-line, o que facilitaria, ainda mais, o acesso, sem a necessidade de reabertura das faculdades e retorno das aulas presenciais para a criação ou manutenção dos núcleos de apoio.

Ressalta-se ainda a necessidade de uma avaliação contínua por meio de estudos e inquéritos para um acompanhamento e para tomada de decisões. Diante disso, é imprescindível avaliar as medidas utilizadas como estratégias 
para uma maior adaptação a este momento de "incertezas", tomando experiências exitosas como exemplo e dados científicos para a elaboração de medidas voltadas ao futuro da educação médica e ao bem-estar biopsicossocial dos discentes de Medicina.

\section{CONTRIBUIÇÃO DOS AUTORES}

Bráulio Brandão Rodrigues, Rhaissa Rosa de Jesus Cardoso, Caio Henrique Rezio Peres e Fábio Ferreira Marques contribuíram suficientemente na concepção e no desenho deste estudo, na análise e interpretação dos dados, bem como na redação deste texto, para que pudessem assumir a autoria e responsabilidade pública pelo conteúdo do artigo, aprovando sua versão final para envio e publicação.

\section{CONFLITO DE INTERESSES}

Os autores declaram não haver conflito de interesses nesta revisão.

\section{REFERÊNCIAS}

1. Zhang Y, Ma ZF. Impact of the COVID-19 pandemic on mental health and quality of life among local residents in Liaoning Province, China: a crosssectional study. Int J Environ Res Public Health. 2020;17(7):1-12.

2. Zu ZY, Jiang MD, Xu PP, Chen W, Ni QQ, Lu GM, et al. Coronavirus disease 2019 (COVID-19): a perspective from China. Radiology. 2020:e15-e25.

3. World Health Organization. Coronavirus Disease (COVID-19) Dashboard. WHO; 2020 [acesso em 9 ago 2020]. Disponível em: https://covid19.who.int/.

4. Russell TW, Hellewell J, Abbott S, Jarvis $\mathrm{Cl}$, Zandvoort $\mathrm{K}$ van, Ratnayake $\mathrm{R}$, et al. Using a delay-adjusted case fatality ratio to estimate underreporting. Centre for Mathematical Modeling of Infectious Diseases Repository; 2020:1-6.

5. Hossain MM, Sultana A, Purohit N. Mental health outcomes of quarantine and isolation for infection prevention: a systematic umbrella review of the global evidence. Epidemiol Health. 2020;42:1-11.

6. Brooks SK, Webster RK, Smith LE, Woodland L, Wessely S, Greenberg N, et al. The psychological impact of quarantine and how to reduce it: rapid review of the evidence. The Lancet. 2020;395:912-20.

7. Wang C, Pan R, Wan X, Tan Y, Xu L, Ho CS, et al. Immediate psychological responses and associated factors during the initial stage of the 2019 coronavirus disease (COVID-19) epidemic among the general population in China. Int J Environ Res Public Health. 2020;17(5):1-25.

8. Meo SA, Abukhalaf D, Alomar AA, Sattar K, Klonoff D. COVID-19 pandemic: impact of quarantine on medical students' mental wellbeing and learning behaviors. Pak J Med Sci. 2020;36(S4):S43-S48.

9. Liu S, Yang L, Zhang C, Xiang YT, Liu Z, Hu S, et al. Online mental health services in China during the COVID-19 outbreak. Lancet Psychiatry. 2020;7(4):e17-e18.

10. Martins RX. A COVID-19 e o fim da educação a distância: um ensaio. EmRede: Revista de Educação a Distância. 2020;7(1):242-56.

11. Arruda EP. Educação remota emergencial: elementos para políticas públicas na educação brasileira em tempos de Covid-19. EmRede: Revista de Educação a Distância. 2020;7(1): 257-75.

12. Araújo FJO, de Lima LSA, Cidade PIM, Nobre CB, Rolim Neto ML. Impact of Sars-CoV-2 and its reverberation in global higher education and mental health. Psychiatry Res. 2020; 288:1-2.
13. Burki TK. COVID-19: consequences for higher education. Lancet Oncol. 2020;21(6):758.

14. Odriozola-González P, Planchuelo-Gómez Á, Irurtia MJ, Luis-García R de. Psychological effects of the COVID-19 outbreak and lockdown among students and workers of a Spanish university. Psychiatry Res. 2020;290:1-8.

15. Brooks SK, Webster RK, Smith LE, Woodland L, Wessely S, Greenberg N, et al. The psychological impact of quarantine and how to reduce it: rapid review of the evidence. The Lancet. 2020;395:912-20.

16. Chang J, Yuan Y, Wang D. Mental health status and its influencing factors among college students during the epidemic of COVID-19. Journal of Southern Medical University. 2020; 40(2):171-176.

17. Zhai $Y$, Du X. Mental health care for international Chinese students affected by the COVID-19 outbreak. Lancet Psychiatry. 2020;7(4):e22.

18. Tang W, Hu T, Hu B, Jin C, Wang G, Xie C et al. Prevalence and Correlates of PTSD and depressive symptoms one month after the outbreak of the COVID-19 epidemic in a sample of home-quarantined Chinese university students. J Affect Disord. 2020;274:1-7.

19. Auerbach RP, Mortier P, Bruffaerts R, Alonso J, Benjet C, Cuijpers P. WHO World Mental Health Surveys International College Student Project: prevalence and distribution of mental disorders. J Abnorm Psychol. 2018;127:623-68.

20. Husky MM, Kovess-Masfety V, Swendsen JD. Stress and anxiety among university students in France during Covid-19 mandatory confinement. Compr Psychiatry. 2020;102: 1-3.

21. Pan X, Chen D, Xia Y, Wu X, Li T, Ou X, et al. Asymptomatic cases in a family cluster with SARS-CoV-2 infection. Lancet Infect Dis. 2020;20(4):410-411.

22. Liu J, Zhu Q, Fan W, Makamure J, Zheng C, Wang J. Online mental health survey in a medical college in China during the COVID 19 outbreak. Frontiers in Psychiatry. 2020;11:1-6.

23. Cao W, Fang Z, Hou G, Han M, Xu X, Dong J, et al. The psychological impact of the COVID-19 epidemic on college students in China. Psychiatry Res. 2020;287:1-5.

24. Akers A, Blough C, Iyer MS. COVID-19 Implications on clinical clerkships and the residency application process for medical students. Cureus. 2020;12(4):1-6.

25. Ferrel MN, Ryan JJ. The impact of COVID-19 on medical education. Cureus. 2020;12(3):1-4.

26. Ahmed H, Allaf M, Elghazaly H. COVID-19 and medical education. Lancet Infect Dis. 2020; 20(7):777-8.

27. Rose S. Medical student education in the time of COVID-19. JAMA. 2020;323(21):2131-2.

28. Anderson ML, Turbow S, Willgerodt MA, Ruhnke GW. Educação em crise: a oportunidade de nossas vidas. J Hosp Med. 2020;15(5):287-91.

29. Qarajeh R, Tahboub F, Rafie N, Pirani N, Jackson MA, Cochran CD. The effect of COVID-19 pandemic on US medical students in US clinical years. International Journal of Medical Students. 2020;8(2):1-6.

30. Alea LA, Fabrea MF, Roldan RDA, Farooqi AZ. Teachers' Covid-19 awareness, distance learning education experiences and perceptions towards institutional readiness and challenges. International Journal of Learning, Teaching and Educational Research. 2020; 19(6):127-44.

31. Sahu P. Closure of universities due to coronavirus disease 2019 (COVID-19): impact on education and mental health of students and academic staff. Cureus. 2020;12(4):e7541.

32. Raaheim A, Mathiassen K, Moen V, Lona I, Gynnild V, Bunæs BR, et al. Digital assessment - how does it challenge local practices and national law? A Norwegian case study. European Journal of Higher Education. 2019;9(2):219-31.

This is an Open Access article distributed under the terms of the Creative Commons Attribution License, which permits unrestricted use, distribution, and reproduction in any medium, provided the original work is properly cited. 